Original Research

\title{
Gymnastic Training and Dynamic Mobilization Exercises Improve Stride Quality and Increase Epaxial Muscle Size in Therapy Horses
}

\author{
Kátia de Oliveira ${ }^{\mathrm{a}}$, Ricardo V.G. Soutello ${ }^{\mathrm{a}}$, Ricardo da Fonseca ${ }^{\mathrm{a}}$, Ciniro Costa ${ }^{\mathrm{b}}$, \\ Paulo R. de L. Meirelles ${ }^{b}$, Daniele F. Fachiolli ${ }^{a}$, Hilary M. Clayton ${ }^{\mathrm{c}, *}$ \\ ${ }^{a}$ College of Animal Science, Experimental Campus of Dracena, Universidade Estadual Paulista “Julio de Mesquita Filho" (UNESP), Rod Cmte. João Ribeiro de Barros, \\ Dracena, São Paulo, Brazil \\ ${ }^{\mathrm{b}}$ Department of Animal Breeding and Nutrition (DABN), Faculty of Veterinary Medicine and Animal Science, Universidade Estadual Paulista "Julio de Mesquita \\ Filho", Botucatu, São Paulo, Brazil \\ ${ }^{\mathrm{c}}$ Sport Horse Science, LLC, Mason, MI
}

\section{A R T I C L E I N F O}

\section{Article history:}

Received 6 June 2015

Received in revised form 4 August 2015

Accepted 4 August 2015

Available online 8 August 2015

\section{Keywords:}

Equine

Hippotherapy

Therapeutic exercise

Dynamic mobilization exercise

Physical training

Three-dimensional movement

\begin{abstract}
A B S T R A C T
The objective was to evaluate the efficacy of gymnastic training (GYM) and dynamic mobilization exercises (DMEs) on stride length (SL) and epaxial muscle size in therapy horses. Nine cross-bred hippotherapy horses that performed three, 25-minute therapeutic riding sessions per week throughout the study period were randomly assigned to three experimental groups: a control group in which the horses were sedentary with no additional physical activity; a group that performed DMEs; and a group that performed both DMEs and additional GYM including pelvic tilting, backing, turning in small circles, and walking over a raised rail to strengthen the abdominal and pelvic stabilizer muscles. The exercises were performed 3 days per week for 3 months, with evaluations at the start and end of the study. Stride quality was assessed by measuring SL and tracking distance (TD). Epaxial muscle size was monitored by ultrasonographic measurement of $\mathrm{m}$. longissimus dorsi (LD) thickness and m. multifidi (MM) cross-sectional area. Paired $t$ tests were used to compare within groups across time, and between groups were detected using analysis of variance with Tukey post hoc test. When walking at $1.3 \mathrm{~m} / \mathrm{s}, \mathrm{SL}$ and TD at walk increased significantly $(P<.05)$ in horses subjected to GYM. Thickness of LD did not change in any group, but cross-sectional area of MM increased significantly by $3.55 \mathrm{~cm}^{2}$ (DME) and $3.78 \mathrm{~cm}^{2}$ (GYM). It was concluded that GYM training improved stride quality and DMEstimulated MM hypertrophy which has been shown to improve intervertebral joint stability in other species.
\end{abstract}

(c) 2015 Elsevier Inc. All rights reserved.

\section{Introduction}

Hippotherapy uses horses as instruments for practitioners to treat individuals who have disabilities or special needs. These riders are often poorly balanced, poorly coordinated, and overweight [1], all of which increase the

\footnotetext{
* Corresponding author at: Hilary M. Clayton, Sport Horse Science, 3145 Sandhill Road, Mason, MI 48854.

E-mail address: claytonh@cvm.msu.edu (H.M. Clayton).
}

stress on the therapy horses. It is not surprising, therefore, that therapy horses are susceptible to work-related injuries which may involve muscular pain [1,2]. It is speculated that these equine injuries may be similar to the muscular pain experienced by human laborers, who, due to overloading or repetitive movements combined with a lack of alternative types of exercise, become susceptible to obesity, back pain, and articular mobility problems [3]. Lumbar pain interferes with the horse's stride quality [4] which has been shown to deteriorate when the riders present special characteristics, 
such as pelvic misalignment and excessive body weight [1]. We hypothesize that poor stride quality could be improved if the horses performed additional exercises specifically designed to recruit and strengthen the core stabilizing and locomotor musculature while moving the joints through a wide range of motion.

One of the benefits of therapeutic riding is that the three-dimensional (3D) movements of the rider's pelvis show similar trajectories and displacement amplitudes when riding a horse at walk to those of an able-bodied person walking overground [5], which stimulates patients to activate their core musculature and stabilize their trunk in the same way as they would if they were walking. The horse's stride quality contributes to the value of the therapeutic experience so it is important for therapy horses to move freely and without restriction of their range of motion [6]. Thus, the therapy horse should have a full range of $3 \mathrm{D}$ movement in order for the patient to receive a quality treatment that accelerates the therapeutic and rehabilitative process. According to Dvorakova et al [7], the combined action of the horse's ventral (hypaxial) and dorsal (epaxial) musculature influences their 3D movement patterns, so specific exercises should be performed by therapy horses with the objective of strengthening the abdominal, sublumbar, and epaxial muscles. The pelvic stabilizer muscles, which maintain correct alignment of the sacroiliac, hip and stifle joints, are necessary for transmission of locomotor forces generated by the hind limb to the horse's trunk [8].

It is well known in people that gymnastic training (GYM) involving muscular stretching and/or strengthening exercises contributes to the prevention of occupational diseases and enhances rehabilitation from injuries [9]. Muscular stretching performed before athletic activity reduces the risk of muscular strain although muscular strength and power may be impaired [10]. Strength training not only improves muscular force and power, it also protects against injury by activating and strengthening the deep stabilizing musculature [9]. In horses, most conditioning studies have focused on cardiovascular fitness in specific sports and relatively little scientific information is available describing the effects of stretching or strength training exercises. Dynamic mobilization exercises (DMEs) involve voluntary movements of the horse's cervical and thoracolumbar intervertebral joints through a wide range of motion with the primary objectives of activating and strengthening the muscles that move and stabilize the intervertebral joints [8]. The kinematic effects of these exercises have been described [11,12], and their value for increasing the cross-sectional area of the equine spinal deep stabilizing $\mathrm{m}$. multifidi (MM) has been reported $[13,14]$. These exercises may also recruit and strengthen the abdominal and pelvic stability muscles although the latter effects have not been evaluated.

Exercises that are thought to play a role in strengthening the abdominal and sublumbar muscles include caudal tilting of the pelvis, stepping backward, and turning in small circles around a barrel [15]. It has been suggested that the pelvic-stabilizing muscles, which include the gluteal muscles, $\mathrm{m}$. biceps femoris, and other hamstring muscles, can be strengthened by stepping over obstacles at walk $[15,16]$.
The objective of this study was to evaluate the effects of DMEs and GYM in therapy horses. The experimental hypotheses are that the regular performance of DMEs will be associated with epaxial muscle hypertrophy and the regular performance of GYM exercises will improve stride quality.

\section{Materials and Methods}

This study was approved by the Ethical Committee for the Use of Animals, of the Animal Husbandry Course, São Paulo State University, Dracena Campus, Brazil, under protocol number 36/2012, in accordance with the ethical principles of animal experimentation.

\subsection{Experimental Design}

The study was conducted in the Association of Parents and Friends of Exceptional Children Hippotherapy Center located in the town of Dracena, São Paulo. The inclusion criteria for horses were that they showed no overt lameness or signs of musculoskeletal lesions during clinical examination, they had been used in hippotherapy sessions for at least 3 years, and they were in regular work. This selection resulted in the inclusion of nine cross-breed therapy horses, with mean age of $16 \pm 3.4$ years and mean body weight of $450 \pm 20.96 \mathrm{~kg}$. The horses were randomly assigned to three groups: sedentary (SED), DMEs, and DMEs plus GYM.

The duration of the experiment was 3 months, during which time the horses continued to be used in hippotherapy sessions three times per week on alternate days with each session being approximately 25 minutes in duration. In addition, the horses performed the designated exercises three times per week under the supervision of a trained professional, who guided the horses using a halter. The SED group did not perform any additional type of physical activity. The DME group performed a series of baited stretches that have been shown to activate and strengthen MM which is a deep spinal-stabilizing muscle [13]. Specifically, the DME exercise routine consisted of three cervical flexion exercises (chin to chest, chin between carpi, and chin to fore fetlocks), a cervical extension exercise and three lateral cervical bending exercises performed to the right and left sides (chin to shoulder, chin to flank, and chin to hind fetlock). Each of the 10 mobilization exercises was repeated five times per exercise session using treats to entice the horses to the desired positions which were then maintained for 5 seconds. Horses were deemed to have performed each exercise successfully when they achieved the position described by Stubbs et al [13] and maintained that position for 5 seconds. The GYM group performed DMEs as described for the DME group together with exercises to recruit and strengthen the abdominal muscles and the pelvic-stabilizing muscles. These exercises were pelvic tilting, backing up, walking around tight turns, and stepping over obstacles at walk. Pelvic tilting was performed at the halt by applying pressure to a point located between $\mathrm{m}$. biceps femoris and $\mathrm{m}$. semitendinosus [8]. Horses were required to hold the pelvic tilt for $5 \mathrm{sec}$ onds with five repetitions per session. Backing up was 
performed in a single series of 10 consecutive steps in a straight line. Tight turns involved walking the horses in small circles around a barrel with three repetitions being performed to the left side and to the right side per session. The obstacle exercise was performed by walking repeatedly over a pole raised to a height of $40 \mathrm{~cm}$ for a total of $10 \mathrm{mi}-$ nutes which included equal amounts of time turning in clockwise and counter-clockwise directions.

The experimental variables were recorded during two evaluations: The initial evaluation was performed before the beginning of the exercise period (day 0), and the final evaluation was conducted after performing the designated activities for 3 months (day 90).

\subsection{Kinematic Measurements}

Linear stride kinematics were evaluated by measuring stride length (SL) and tracking distance (TD) as indicators of stride quality and to verify hind limb engagement. To perform the kinematic evaluations, a lane $8 \mathrm{~m}$ long and $2 \mathrm{~m}$ wide was constructed on a sand track. Before the evaluations, the horses were warmed up at walk in hand for 15 minutes and then taken to the sand lane for measurement [4]. The horses passed through the lane three times at walk, at a target speed of $1.3 \mathrm{~m} / \mathrm{s}$. A photocell was used to monitor speed and to remove trials that differed from the target speed [4]. The horses' movements were captured at $25 \mathrm{~Hz}$ by a video camera oriented perpendicular to and $8 \mathrm{~m}$ from the left side of the evaluation track [17]. From these trials, three strides per horse were selected for analysis on the basis of being performed with a consistent speed and rhythm [18].

Video images were transferred to a computer for determination of linear measurements by a single operator using a previously validated technique. The runway was calibrated using marker poles, and SL was measured as the distance between successive ground contacts of the left forelimb. The TD was the distance between the hoof prints of the left hind hoof and left fore hoof with the value being designated positive if the hind hoof stepped ahead of the fore hoof (overtracking), negative if the hind hoof stepped behind the fore hoof (undertracking), or zero if the hind hoof stepped into the fore hoof print (tracking up) [19].

\subsection{Biometric Evaluations}

Biometric evaluations of the muscles involved ultrasonographic measurements of the thickness of longissimus dorsi (LD) and the cross-sectional area of MM, to monitor epaxial musculature development. These evaluations were performed using a PIE MEDICAL Scanner 200 VET (Pie Medical Imaging BV, Maastricht, the Netherlands) in real time, with a 3.5-MHz transducer $13 \mathrm{~cm}$ in length. All ultrasonographic images were taken on the horse's left side by the same qualified professional who was blinded to the horse and the treatment group assignments. The LD measurement was performed on the left side at the level of the two last ribs with the probe placement as described by D'Angelis et al [20]. The horse stood with the forelimb pair and the hind limb pair aligned and with the LD muscle relaxed. Three images were obtained and used to measure the thickness of the LD muscle. The MM images were obtained at the level of the fifth lumbar vertebra. The lumbosacral junction was identified by the divergence of the spinous processes of the last lumbar (L6) and first sacral (S1) vertebrae, and the spine of the fifth lumbar vertebra (L5) was located cranial to L6. Three ultrasound images were acquired between the cranial and caudal articular processes of $\mathrm{L} 6$ with the ultrasound probe adjacent to the dorsal midline, oriented transversely, and held at an angle of approximately $45^{\circ}$. Landmarks were medially the bony margin of the dorsal spinous process, ventrally the bony margin of the rib or transverse process, and laterally the fascial border between MM and LD. Cross-sectional area of MM was determined by tracing around the periphery of the MM as described by Stubbs et al [13]. Determination of LD thickness and MM cross-sectional area was obtained using the manufacturer's software.

\subsection{Statistical Analysis}

Statistical analyses were performed using SAS (SAS Institute Inc, Cary, NC, USA). Mean values and standard deviations were calculated for each variable describing the linear kinematics and muscular biometry. The Kolmogorov-Smirnov test indicated that the variables were normally distributed. Initial and final values for each variable were compared using paired $t$ tests. Analysis of variance was used to detect differences between mean values for the three groups, and when significant differences were detected, post hoc Tukey tests were used to determine which groups differed from each other. All statistical tests used a $5 \%$ probability.

\section{Results}

Mean walking speed $(1.3 \mathrm{~m} / \mathrm{s})$ did not differ between the initial and final evaluations. Over the 3-month study period, SL increased significantly in the GYM group by $10.67 \pm 2.08 \mathrm{~cm}$, and at the final evaluation, SL was significantly longer in the GYM group compared with the SED or DME groups (Table 1). At the initial evaluation, TD was negative in all three groups. The value increased significantly only in the GYM group which showed an increase of $16.73 \pm 2.20 \mathrm{~cm}$. On average, horses in the GYM group overtracked by $5.30 \pm 1.0 \mathrm{~cm}$ at the final evaluation and TD was significantly longer in this group than in SED or DME groups $(P=.0004)$ (Table 2$)$.

The thickness of the LD muscle did not change significantly between the initial and final evaluations in any of the groups and did not differ across groups (Table 1). Crosssectional area of MM increased significantly from the initial to the final evaluation in both groups that performed DMEs. The measured increases were $3.55 \mathrm{~cm}^{2}$ in the DME group and $3.78 \mathrm{~cm}^{2}$ in the GYM group. At the final evaluation, these two groups did not differ from each other, but both had a significantly larger cross-sectional area than the SED group $(P=.004)$ (Table 2$)$.

\section{Discussion}

The results of our study have shown that GYM was associated with increases in both SL and TD at a controlled 
Table 1

Initial and final measurements for variables describing stride quality and epaxial muscle size in therapy horses performing different exercise protocols.

\begin{tabular}{|c|c|c|c|c|c|c|c|}
\hline \multirow[t]{2}{*}{ Variable } & \multirow[t]{2}{*}{ Condition } & \multicolumn{3}{|l|}{ Treatment } & \multicolumn{3}{|c|}{ Probability } \\
\hline & & SED & DME & GYM & SED & DME & GYM \\
\hline \multirow[t]{2}{*}{ Stride length $(\mathrm{cm})$} & Initial & $126.00 \pm 7.21$ & $137.33 \pm 17.21$ & $146.00 \pm 4.35$ & 0.9225 & 0.8776 & 0.0299 \\
\hline & Final & $126.67 \pm 8.50$ & $139.67 \pm 17.61$ & $156.67 \pm 3.51$ & & & \\
\hline \multirow[t]{2}{*}{ Tracking length $(\mathrm{cm})$} & Initial & $-15.00 \pm 4.00$ & $-8.30 \pm 2.08$ & $-11.43 \pm 1.20$ & 0.6318 & 0.2910 & 0.0001 \\
\hline & Final & $-13.17 \pm 4.65$ & $-6.44 \pm 1.63$ & $5.30 \pm 1.00$ & & & \\
\hline \multirow[t]{2}{*}{ Thickness of muscle longissimus dorsi $(\mathrm{cm})$} & Initial & $5.26 \pm 1.34$ & $5.40 \pm 0.60$ & $4.94 \pm 1.79$ & 0.7632 & 0.3685 & 0.8207 \\
\hline & Final & $5.02 \pm 1.32$ & $5.80 \pm 0.85$ & $5.18 \pm 1.83$ & & & \\
\hline \multirow[t]{2}{*}{ Cross-sectional area of muscle multifidi $\left(\mathrm{cm}^{2}\right)$} & Initial & $7.57 \pm 2.11$ & $6.83 \pm 2.30$ & $7.08 \pm 1.34$ & 0.6834 & 0.0330 & 0.0138 \\
\hline & Final & $7.02 \pm 2.40$ & $10.38 \pm 2.66$ & $10.86 \pm 2.80$ & & & \\
\hline
\end{tabular}

Abbreviations: DME, dynamic mobilization exercises performed 3 days per week; GYM, dynamic mobilization exercises and gymnastic exercises performed 3 days per week; SD, standard deviation; SED, sedentary group, no additional exercise.

Values are mean $\pm \mathrm{SD}$.

Values in italics indicate significant differences within groups between initial and final measurements, and statistical probabilities are shown in the columns on the right.

walking speed which is interpreted as indicating improved gait quality and is likely to improve the therapeutic benefits to the rider. Additionally, horses that performed DMEs showed significant increases in cross-sectional area of the deep spinal stabilizer MM which is potentially beneficial in preventing micromotion or instability of the intervertebral joints during locomotion and thus protecting the horses against the development of facet joint osteoarthritis. These findings support the experimental hypotheses.

The horses' movements were evaluated at the walk because this is the gait used most frequently in therapy sessions. In the current research, the SL at walk was $136 \pm$ $9.6 \mathrm{~cm}$ in therapy horses walking at $1.3 \mathrm{~m} / \mathrm{s}$ which is similar to the SL of 128 to $132 \mathrm{~cm}$ for speeds in the range of 1.35 to $1.43 \mathrm{~m} / \mathrm{s}$ reported for therapy horses by Dvorakova et al [7]. As might be expected, SL values in therapy horses are lower than those reported for taller warmblood sport horses in which SL was $157 \mathrm{~cm}$ for a collected walk at $1.37 \mathrm{~m} / \mathrm{s}$ [21].

Because the walk does not have a suspension phase, alterations in SL are dependent primarily on changes in limb protraction and retraction, which are determined by movements of the proximal joints augmented by flexion/ extension of the thoracolumbar spine and lumbosacral joint. Changes in TD are predictive of alterations in SL in all gaits [19]. In order for the hind limb to step forward and overtrack the forelimb at the walk, the epaxial muscles must be relaxed so that the thoracolumbar spine can be rounded by the bow and string mechanism [22]. Activity of $\mathrm{m}$. rectus abdominis and the external abdominal oblique muscle is significantly and positively correlated with hind limb protraction [23], which supports a contribution of the abdominal muscle strengthening exercises in the GYM training to the significant increase in TD. The gymnastic exercises chosen for the study would be expected to increase spinal flexibility, strengthen the abdominal musculature, improve range of motion of the joints of the limbs, and strengthen the abductor and adductor muscles. Specifically, the pelvic tilting and backing exercises activate and strengthen the muscles that flex the thoracolumbar spine and lumbosacral joint.

Hip flexion and extension are the main determinants of hind limb protraction and retraction, respectively, while in the forelimb flexion and extension of the elbow joint, assisted by rotation and translation of the scapula across the chest wall determine the range of protraction and retraction. Trotting over poles up to $20 \mathrm{~cm}$ high is associated with significant increases in swing phase flexions of all joints of both the fore and hind limbs [24]. In the study reported here, walking over poles at a height of $40 \mathrm{~cm}$ was likely to require even greater flexion of the joints in order for the hooves to clear the pole. Increases in the range of motion of the proximal limb joints are likely to have contributed to the increases in SL and TD. When the joints rotate through a wider range of motion, hind limb protraction increases and contributes to greater engagement of the hind limb. The changes in back and limb kinematics that were responsible for the increase in SL may also intensify the oscillations of the horse's back [1], thereby further improving the therapeutic value of the horse's movement pattern. Another technique not used in the

Table 2

Differences between the initial and final measurements for variables describing stride quality and epaxial muscle size in therapy horses performing different exercise protocols and the probabilities of between-group differences.

\begin{tabular}{lcccc}
\hline Variable & Treatment & & \multirow{2}{*}{ Probability } \\
\cline { 2 - 4 } & SED & DME & GYM \\
\hline Stride length $(\mathrm{cm})$ & $0.67 \pm 1.52^{\mathrm{a}}$ & $2.33 \pm 0.58^{\mathrm{a}}$ & $10.67 \pm 2.08^{\mathrm{b}}$ & 0.0004 \\
Tracking length $(\mathrm{cm})$ & $1.83 \pm 2.75^{\mathrm{a}}$ & $1.86 \pm 0.46^{\mathrm{a}}$ & $16.73 \pm 2.20^{\mathrm{b}}$ & 0.0002 \\
Thickness of muscle longissimus dorsi $(\mathrm{mm})$ & $2.38 \pm 0.91$ & $3.98 \pm 1.63$ & $2.43 \pm 1.08$ & 0.0686 \\
Cross-sectional area of muscle multifidi $\left(\mathrm{cm}^{2}\right)$ & $-0.55 \pm 0.73^{\mathrm{a}}$ & $3.55 \pm 2.69^{\mathrm{b}}$ & $3.78 \pm 2.36^{\mathrm{b}}$ & 0.0043 \\
\hline
\end{tabular}

Abbreviations: DME, dynamic mobilization exercises performed 3 days per week; GYM, dynamic moblization exercises and gymnastic exercises performed 3 days per week; SD, standard deviation; SED, sedentary group, no additional exercise.

Values are mean \pm SD.

Different superscripts indicate values that are significantly different $(P<.05)$. 
present study but that might be useful for increasing hind limb protraction in therapy horses is massage. Hill and Crook [17] reported that massaging the superficial gluteal muscle, $\mathrm{m}$. biceps femoris, and $\mathrm{m}$. semitendinosus significantly increased both passive and active hind limb protraction with horses taking longer trotting stride after massage.

The $\mathrm{m}$. longissimus is the largest and longest muscle in the horse's body. It extends from the sacrum and ilium to the neck and head. It is divided into lumbar, thoracic, cervical, and capital parts. In the thoracolumbar region, where it occupies the area between the dorsal spinous processes and the lumbar transverse process or the dorsal part of the ribs, it is often referred to as LD [25]. In the present study, LD did not show significant hypertrophy indicating that the type, intensity, or duration of physical activity were inadequate to stimulate a significant transformation in this muscle. Similarly, O'Connor et al [26] did not observe LD hypertrophy in horses trained with a $45 \mathrm{~kg}$ (99 lb) weight overload applied to the back, in comparison with conventional training. In addition, Cottrial et al [27] also failed to verify an increase in electromyographic activity in LD in horses trained with the Pessoa rein.

The functions of the LD muscle include provision of stiffness and bending moments to the intervertebral joints. When the LD muscle contracts concentrically, the main effects are to extend the vertebral column when acting bilaterally or cause lateral bending when acting unilaterally [27-32]. During trotting, LD is active during the suspension phase to limit thoracolumbar rounding induced by gravitational, inertial, and locomotor forces [28,32]. Activation increases with speed due to the need for greater spinal stabilization $[23,32]$. Thus, LD activation appears to be more important in gaits that have a suspension phase and especially at faster speeds. The LD muscle also shows increased electromyographic activity during turning [29]. The present study trained horses only at the walk in straight lines; it would probably be necessary to include faster gaits and/or turning exercises to recruit and strengthen LD.

The LD muscle tends to go into spasm in horses with minor back pain or when MM is dysfunctional [33] which may shorten SL and have a negative effect on stride quality by interfering with thoracolumbar flexion. Resting LD muscle tone is influenced by muscular and articular afferent input. Chronic muscular irritation provides continuous feedback that maintains the elevated muscle tone. This is identified as muscle spasm [34] and restriction of movement. However, subclinical tissue abnormalities are often present without perceptible pain although they may exhibit muscle tension and altered ranges of joint motion [34]. The exercises performed by the GYM group recruited the LD muscle, especially during turning [27,30], and also stretched this muscle when the thoracolumbar spine and lumbosacral joint were flexed during the pelvic tilting and rein back exercises.

The MMs are deeply located and not accessible to visual evaluation. The fascicles of MM are short and cross from one to four intervertebral joints in the thoracic, lumbar, and sacral regions [35]. This structure is suitable for opposing transverse and rotational forces exerted on the vertebrae by the abdominal and pelvic muscles [13]. The MMs are thought to act similarly in humans and in horses; in humans, the muscle has been shown to be preactivated in anticipation of movement to provide segmental stabilization and spinal control during locomotion [13,36]. This type of spinal stability is highly relevant in therapy horses due to the perturbations induced by the poor balance and coordination of the patients [1].

The results presented here provide further support for the role of DMEs in activating and strengthening MM leading to a significant increase in cross-sectional area. Similar findings have been reported by Stubbs et al [13] and Tabor [14]. In the study of Stubbs et al [13], SED horses that performed the same series of DME as performed here on 5 days per week for 3 months showed significant MM hypertrophy at six-spinal levels from T10 to L5 and also significant improvements in left: right symmetry at all six levels. At the end of that study, MM cross-sectional area was $11 \mathrm{~cm}^{2}$ [13] which is very similar to the cross-sectional areas of $10.38 \mathrm{~cm}^{2}$ (DME) and $10.62 \mathrm{~cm}^{2}$ (GYM) at the end of our study. This finding implies that performing DME 5 times per week does not provide additional benefit over performing DME three times per week. The addition of GYM exercises to the DME group did not augment MM hypertrophy. In racehorses, Tabor [14] found a significant increase in MM crosssectional area when adding DME to the horse's regular race training regime. These findings indicate that specific exercises, rather than general athletic training, are required to stimulate hypertrophy of MM. The benefits of MM hypertrophy have not been definitively proven in horses; we hypothesize that if this muscle acts to stabilize the intervertebral joints during locomotion, then hypertrophy may improve athletic performance by facilitating transmission of propulsive forces from the hind limbs.

\section{Conclusions}

Our findings show that performing GYM three times per week increased the horse's SL and TD which are considered to be indicators of improved stride quality and may enhance the value of the therapeutic experience for patients. Performance of DMEs three times per week stimulated hypertrophy of MM which has been shown to contribute to stabilization of the intervertebral joints in anticipation of voluntary movement. The inclusion of these exercises in the therapy horse's program is likely to improve both their ability to provide a high-quality therapeutic experience to patients and may have a beneficial effect on their longevity as participants in a therapeutic riding program.

\section{Acknowledgments}

None of the authors has any financial or personal relationships that could inappropriately influence or bias the content of the article.

\section{References}

[1] Rosa LR. Biomechanical analysis of a horse therapy: the interference of body weight and posture of the symmetry of the quality of the 
practitioner step horse. Brasília. In: XII Congresso Brasileiro de Equoterapia; 2006. p. 218-29 (in Portuguese, with abstract in English).

[2] Silva MNG, Folchini NP, Mistieri MLA, Freitas GSR, Sodré LAD, Duarte CA. Survey of disorders occurring in horses used in hippotherapy in the period 2006 to 2010 in Uruguaiana-RS. Revista Brasileira de Ciência Veterinária 2012;19:139-43 (in Portuguese, with abstract in English).

[3] Allender S, Cowburn G, Foster C. Understanding participation in sport and physical activity among children and adults: a review of qualitative studies. Health Education Research 2006;21:826-35.

[4] Janura M, Dvorakova T, Peham C. The influence of walking speed on equine back motion in relation to hippotherapy. Vet Med Austria 2010;97:1-5.

[5] Garner BA, Rigby BR. Human pelvis motions when walking and when riding a therapeutic horse. Hum Mov Sci 2015;39:121-37.

[6] Svoboda Z, Dvořáková T, Janura M. Does the rider influence the horses movement in hippotherapy? Acta Universitatis Palackianae Olomucensis. Gymnica 2011;41:37-41.

[7] Dvorakova T, Janura M, Svoboda Z. The influence of the leader on the movement of the horse in walking during repeated hippotherapy sessions. Acta Universitatis Palackianae Olomucensis. Gymnica 2009;39:43-50.

[8] Stubbs NC, Clayton HM. Activate your horse's core. Mason: Sport Horse Publications; 2008. p. 32.

[9] Barr KP, Griggs M, Cadby T. Lumbar stabilization: core concepts and current literature, Part 1. Am J Phys Med Rehabil 2005;84:473-80.

[10] McHugh MP, Cosgrave $\mathrm{CH}$. To stretch or not to stretch: the role of stretching in injury prevention and performance. Scand J Med Sci Sports 2010;20:169-81.

[11] Clayton HM, Kaiser LJ, Lavagnino M, Stubbs NC. Dynamic mobilizations in cervical flexion: effects on intervertebral angulations, Equine Vet J 2010;42:688-94.

[12] Clayton HM, Kaiser LJ, Lavagnino M, Stubbs NC. Evaluation of intersegmental vertebral motion during performance of dynamic mobilization exercises in cervical lateral bending in horses. Am J Vet Res 2012;73:1153-9.

[13] Stubbs NC, Kaiser LJ, Hauptman J, Clayton HM. Dynamic mobilization exercises increase cross sectional area of musculus multifidus. Equine Vet J 2011;43:522-9.

[14] Tabor GF. The effect of dynamic mobilisation exercises on the equine multifidus muscle and thoracic profile. Masters Thesis. Plymouth, UK: Plymouth University; 2014.

[15] Higgins G. How your horse moves. Cincinnati: David \& Charles Book; 2009.

[16] Higgins G. Pilates and stretching. Cincinnati: Horse Inside Out Publication; 2009.

[17] Hill C, Crook T. The relationship between massage to the equine caudal hindlimb muscles and hindlimb potraction. Equine Vet J 2010;42:638-87.

[18] Schils SJ, Greer NL, Stoner LJ, Kobluk CN. Kinematic analysis of the equestrian-walk, posting trot and sitting trot. Hum Mov Sci 1993; 12:693-712.
[19] Clayton HM. The dynamic horse. Mason: Sport Horse Publications; 2004.

[20] D'angelis FHF, Mota MDS, Freitas EVV, Ferraz GC, Abrahão AR, Lacerda-Neto JC, Queiroz-Neto A. Aerobic training but not creatine supplementation modifies longissimus dorsi muscle composition. J Equine Vet Sci 2007;27:118-22.

[21] Clayton HM. Comparison of the stride kinematics of the collected, medium, and extended walks in horses. Am J Vet Res 1995;56: 849-52.

[22] Waldern NM, Wiestner T, Peinen K. Influence of different head-neck positions on vertical ground reaction forces, linear and time parameters in the unridden horse walking and trotting on a treadmill. Equine Vet J 2009;41:268-73.

[23] Zsoldos RR, Kotschwar A, Kotschwar AB. Activity of equine rectus abdominis and oblique external abdominal muscles measured by surface EMG during walk and trot on the treadmill. Equine Vet J 2010;42(suppl.38):523-9.

[24] Brown S, Stubbs N, Kaiser L, Lavagnino M, Clayton HM. Swing phase kinematics of horses trotting over poles. Equine Vet J 2015;47:107-12.

[25] Getty R. Sisson and Grossman's the anatomy of the domestic animals. 5th ed. Philadelphia: WB Saunders Company; 1975.

[26] O'Connor CI, Nielsen BD, Schott HC. Effects of weight carrying, exercise and a myo-anabolic supplement on growth and muscle. Equine Vet J 2002;34:178-81.

[27] Cottriall S, Ritruechai P, Wakeling JM. The effects of training aids on the longissimus dorsi in the equine back. Comp Exerc Physiol 2009; 3-4:111-4.

[28] McGowan C, Stubbs N, Hodges P, Jeffcott L. Back pain in horses-epaxial musculature. Canberra, Australian Capital Territory: Rural Industries Research and Development Corporation; 2007.

[29] Wakeling JM. Segmental variation in the activity and function of the equine longissimus dorsi muscle during walk and trot. Equine Comp Exerc Physiol 2007;4:95-103.

[30] Wakeling JL, Barnett K, Price S, Nankervis K. Effects of manipulative therapy on the longissimus dorsi in the equine back. Equine Comp Exerc Physiol 2006;3:153-60.

[31] Groesel M, Zsoldos RR, Kotschwar A. A preliminary model study of the equine back including activity of longissimus dorsi. Equine Vet J 2010;42(suppl 38):401-6.

[32] Robert C, Valette JP, Denoix J-M. The effects of treadmill inclination and speed on the activity of three trunk muscles in the trotting horse. Equine Vet J 2001;33:466-72.

[33] Clayton HM. Equine back pain reviewed from a motor control perspective. Comp Exerc Physiol 2012;8:145-52.

[34] Wolf L. The role of complementary techniques in managing musculoskeletal pain in performance horses. Vet Clin Equine 2002; 18:107-15.

[35] Stubbs NC, Hodges PW, Jeffcott LB. Functional anatomy of the caudal thoracolumbar and lumbosacral spine in the horse. Equine Vet J 2006;38:393-9.

[36] Haussler KK, Hill AE, Puttlitz CM. Effects of vertebral mobilization and manipulation on kinematics of the thoracolumbar region. Am J Vet Res 2007;68:508-16. 